\title{
Tetracycline therapy targets intracellular bacteria in the filarial nematode Litomosoides sigmodontis and results in filarial infertility
}

\author{
Achim Hoerauf, ${ }_{1}^{1}$ Kerstin Nissen-Pähle, ${ }^{1}$ Christel Schmetz, ${ }^{1}$ Kim Henkle-Dührsen, ${ }^{1}$ \\ Mark L. Blaxter, ${ }^{2}$ Dietrich W. Büttner, ${ }^{1}$ Michaela Y. Gallin, ${ }^{1}$ Khaled M. Al-Qaoud, ${ }^{1}$ \\ Richard Lucius, ${ }^{3}$ and Bernhard Fleischer ${ }^{1}$
}

${ }^{1}$ Bernhard Nocht Institute for Tropical Medicine, 20359 Hamburg, Germany

${ }^{2}$ Institute of Cell, Animal and Population Biology, University of Edinburgh, Edinburgh E H9 3DT, United Kingdom

${ }^{3}$ Lehrstuhl für Molekulare Parasitologie, Humboldt-Universität, 10115 Berlin, Germany

Address correspondence to: Achim Hoerauf, Bernhard Nocht Institute for Tropical Medicine, Bernhard-Nocht-Strasse

74, 20359 Hamburg, Germany. Phone: 49-40-31182-495; Fax: 49-40-31182-400; E-mail: hoerauf@bni.uni-hamburg.de

Received for publication August 1, 1998, and accepted in revised form October 22, 1998.

\begin{abstract}
Intracellular bacteria have been described in several species of filarial nematodes, but their relationships with, and effects on, their nematode hosts have not previously been elucidated. In this study, intracellular bacteria were observed in tissues of the rodent parasite Litomosoides sigmodontis by transmission electron microscopy and by immunohistochemistry using antiendobacterial heat shock protein-60 antisera. Molecular phylogenetic analysis of the bacterial 16S ribosomal RNA gene, isolated by PCR, showed a close relationship to the rickettsial Wolbachia endobacteria of arthropods and to other filarial intracellular bacteria. The impact of tetracycline therapy of infected rodents on $L$. sigmodontis development was analyzed in order to understand the role(s) these bacteria might play in filarial biology. Tetracycline therapy, when initiated with $L$. sigmodontis infection, eliminated the bacteria and resulted in filarial growth retardation and infertility. If initiated after microfilarial development, treatment reduced filarial fertility. Treatment with antibiotics not affecting rickettsial bacteria did not inhibit filarial development. Acanthocheilonema viteae filariae were shown to lack intracellular bacteria and to be insensitive to tetracycline. These results suggest a mutualistic interaction between the intracellular bacteria and the filarial nematode. Investigation of such a mutualism in endobacteria-containing human filariae is warranted for a potential chemotherapeutic exploitation.
\end{abstract}

J. Clin. Invest. 103:11-18 (1999)

\section{Introduction}

Infections with filarial nematodes affect more than 150 million people worldwide, causing a variety of symptoms from mild dermatitis to blindness and debilitating forms of elephantiasis. The world community has therefore made it a goal to eliminate these diseases (1). However, current drug treatments are targeted at elimination of the larval microfilariae circulating in the bloodstream or in the skin, and not against the adult worms. Because adults can live for up to 15 years, and microfilarial loads rise again after treatment, it will be necessary to maintain mass treatment for many years if the cycle of transmission is to be interrupted. There is thus a pressing need for drugs active against adult filariae (macrofilaricidal or embryostatic) in order to complement microfilaricides such as ivermectin (2), which has been documented to act only against late stages of microfilarial development (3). In addition, genetic resistance to two of the current filaricides (ivermectin and albendazole) has been documented in intestinal nematodes parasitizing livestock (4). Although the generation and maintenance of resistance in the filariae is likely to be much less common, experts still see it as a necessity to explore new avenues for drug and vaccine design (2).
Intracellular bacteria were observed in human- and animal-parasitic filarial nematodes some 20 years ago (5-8). These bacteria appeared to be vertically transmitted by inclusion with the oocyte during development, and infection was highly prevalent in species carrying the bacteria. Few species of filariae (e.g., Acanthocheilonema viteae) did not appear to harbor bacteria (5). Molecular phylogenetic analysis has recently shown that the intracellular bacteria of Dirofilaria immitis, the dog heartworm, are closely related to the cytoplasmic incompatibility endobacteria of arthropods, of the genus Wolbachia (order Rickettsiales) (9). Members of the genus Wolbachia are found in $20 \%$ of arthropod species (10). They are sometimes termed endosymbionts, a term that may be considered with regard to the original definition of "symbiosis" not in functional but in structural terms (based on intimate association with hosts) (11). However, there are examples of both mutualism and parasitism (12), and pathogenic strains of arthropod Wolbachia have been described (13).

The biology of the interrelationship between filarial endobacteria and their nematode hosts remains to be elucidated. Early workers hypothesized a pathogenic effect, because some embryos appeared distorted by the number of bacteria they carried (5). This was not proved, however, 
Table 1

Peak serum concentrations of antibiotics

\begin{tabular}{lccc}
\hline Antibiotic & $\begin{array}{c}\text { Dose } \\
(\mathrm{mg} / \mathrm{kg} / \text { day })\end{array}$ & $\begin{array}{c}\text { Peak serum concentration } \\
(\mu \mathrm{g} / \mathrm{ml})\end{array}$ & $\begin{array}{c}\mathrm{MIC}^{\mathrm{B}} \\
(\mu \mathrm{g} / \mathrm{ml})\end{array}$ \\
Tetracycline & 50.0 & $8.3 \pm 0.8$ & 4 \\
& 25.0 & $5.0 \pm 1.1$ & 4 \\
Gentamicin & 12.5 & $3.9 \pm 1.0$ & 4 \\
Penicillin G & 15.0 & $14.0 \pm 2.8$ & 4 \\
Ciprofloxacin & 500.0 & $53.0 \pm 9.4$ & 0.1 \\
\hline
\end{tabular}

${ }^{A}$ Determined $15-30 \mathrm{~min}$ after intraperitoneal application of antibiotic.

BMinimal inhibitory concentration for susceptible microorganisms according to the National Committee for Clinical Laboratory Standards (33).

and it remains possible that the association is neutral (commensal) or even beneficial (mutualistic). The vertical mode of transmission would tend to promote the evolution of mutualistic associations $(14,15)$. We have examined this aspect of the interaction by using antibiotics predicted to affect specifically the rickettsial endobacterium in the nematode host. A strong antihelminthic effect of tetracycline against bacteria-containing filariae and lack of such effects in a bacteria-free filarial species argue for a mutualistic interaction. The prokaryote genome of this endosymbiont offers a wide range of specific targets that might be exploited for the treatment of infections with human-pathogenic filariae that also contain endobacteria, such as Onchocerca volvulus (6) and Brugia malayi (7).

\section{Methods}

Rodents. The following rodents were bred in the animal facilities of the Bernhard Nocht Institute: (a) BALB/c mice (inbred strain of Mus musculus [family Muridae, order Rodentia]); (b) multimammate rats (Mastomys coucha [ref. 16; family Muridae, order Rodentia]); (c) jirds (Meriones unguiculatus [family Cricetidae, order Rodentia]); and (d) cotton rats (Sigmodon bispidus [family Cricetidae, order Rodentia]). The evolutionary relationship of these rodent species can be inferred from their zoologic classification (17). Among the approximately 30 families within the order of Rodentia, suborder Myomorpha, the families of Muridae and Cricetidae are most closely related; however, there is a closer relationship between BALB/c mice and multimammate rats on the one hand (this family also includes Rattus rattus, the normal laboratory rat) and between jirds and cotton rats on the other hand. In this study, cotton rats and jirds, being the natural hosts of Litomosoides sigmodontis and $A$. viteae filariae, respectively, were used for maintenance of the parasites, which cannot be cultured in vitro, whereas mice and multimammate rats (and cotton rats in the experiments for Fig. 4) were used for infection and antibiotic treatment experiments. Rodents were infected at an age of 6-8 weeks.

Parasite maintenance. L. sigmodontis filariae were maintained by passage through cotton rats, as described previously $(18,19)$. Infection was achieved by first feeding mites (Ornithonyssus bacoti) on microfilaremic $(>2,000 \mathrm{mf} / \mu \mathrm{l}$ blood) cotton rats. Ten days later (i.e., after maturation of infectious stage 3 larvae in the mites), the mites were allowed to feed on the rodents to be infected for experimental purposes (BALB/c, multimammate rats, and cotton rats), thereby transmitting third-stage larvae (L3). To ensure comparability, animals treated and not treated with antibiotics were always exposed to the same population of mites.

The life cycle of $A$. viteae was maintained in jirds and soft ticks (Ornithodoros moubata) (20). For infection experiments, L3 were isolated from dissected ticks 6 weeks after the ticks had been fed on microfilaremic jirds. L3 were rinsed in Roswell Park Memorial Institute medium and $80 \mathrm{~L} 3$ per animal were injected subcutaneously into multimammate rats.

Antibiotic treatment. Tetracycline hydrochloride was obtained from Boehringer Mannheim (Mannheim, Germany) and dissolved at $5 \mathrm{mg} / \mathrm{ml}$ in PBS. Gentamicin, penicillin, and ciprofloxacin in fluid form were purchased from the hospital pharmacy serving the Bernhard Nocht Institute.

$\mathrm{BALB} / \mathrm{c}$ mice received daily intraperitoneal injections with the above antibiotics using the following doses per kilogram of body weight (average mouse body weight: $20 \mathrm{~g}$ ): tetracycline: 50, 25, or $12 \mathrm{mg} / \mathrm{kg}$, according to the experiment; gentamicin: 15 $\mathrm{mg} / \mathrm{kg}$; penicillin: $600 \mathrm{mg} / \mathrm{kg}$; ciprofloxacin: $50 \mathrm{mg} / \mathrm{kg}$. Multimammate rats also received daily intraperitoneal injections with tetracycline $(25 \mathrm{mg} / \mathrm{kg})$. BALB/c mice and multimammate rats received treatment from day 1 after infection until the end of the respective experiment. Cotton rats were given tetracycline at $0.3 \%$ in drinking water from days 150 to 191 after infection. To determine the peak concentration of antibiotics in the serum of animals, blood was drawn 15-30 min after injection of the antibiotic, and serum antibiotic levels were determined by agar diffusion technique (21). Serial dilutions of sera (1:5-1:50 in PBS) were generated, and $10 \mu \mathrm{l}$ of each dilution was pipetted (with the help of a 5-mm-diameter cylinder) on an agar plate containing $10^{5}$ Bacillus subtilis $\mathrm{CFU} / \mathrm{ml}$ agar. Ten-microliter serial dilutions $(5-0.1 \mu \mathrm{g} / \mathrm{ml})$ of stock solutions of each antibiotic to be tested were pipetted in the same way on these agars, and plates were incubated overnight at $37^{\circ} \mathrm{C}$ to allow bacterial growth. Thereafter, the diameter of the growth inhibition zone around the inoculation spots of the antibiotic standards was
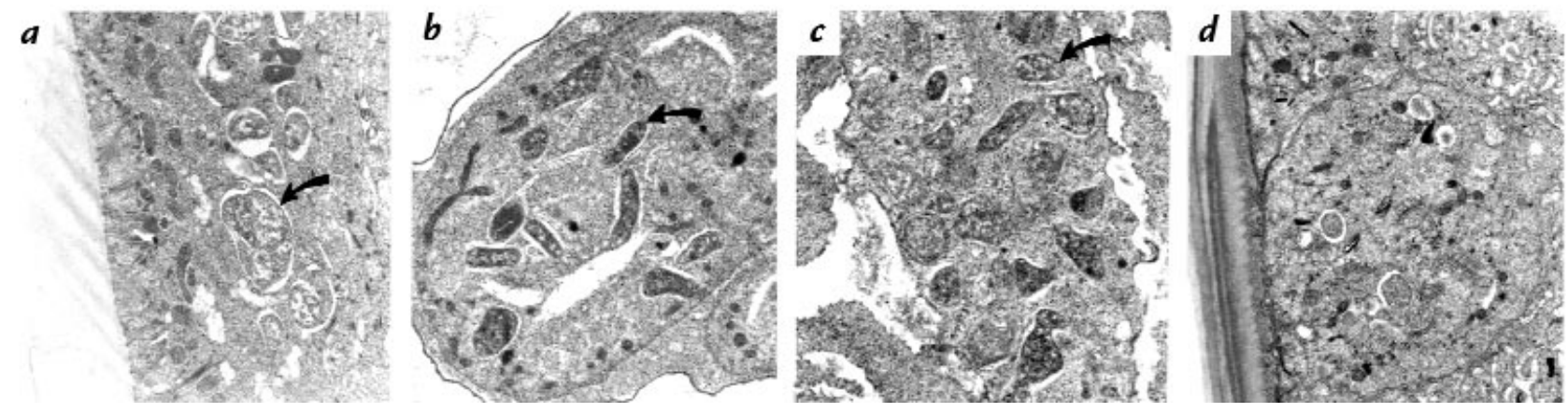

\section{Figure 1}

Electron microscopy of female Litomosoides sigmodontis from BALB/c mice with or without tetracycline therapy. (a-c) Worms from untreated mouse (day 28 after infection). Bacteria (arrows) are prominent in the lateral chords of the hypodermis (a), in the rachis of the ovary (b), and in embryonic stages within the uterus $(\boldsymbol{c}) .(\boldsymbol{d})$ Worm from tetracycline-treated mouse (day 28 after infection). Remnants of damaged bacteria (arrow) are seen in the hypodermis. $\times 14,500$. 


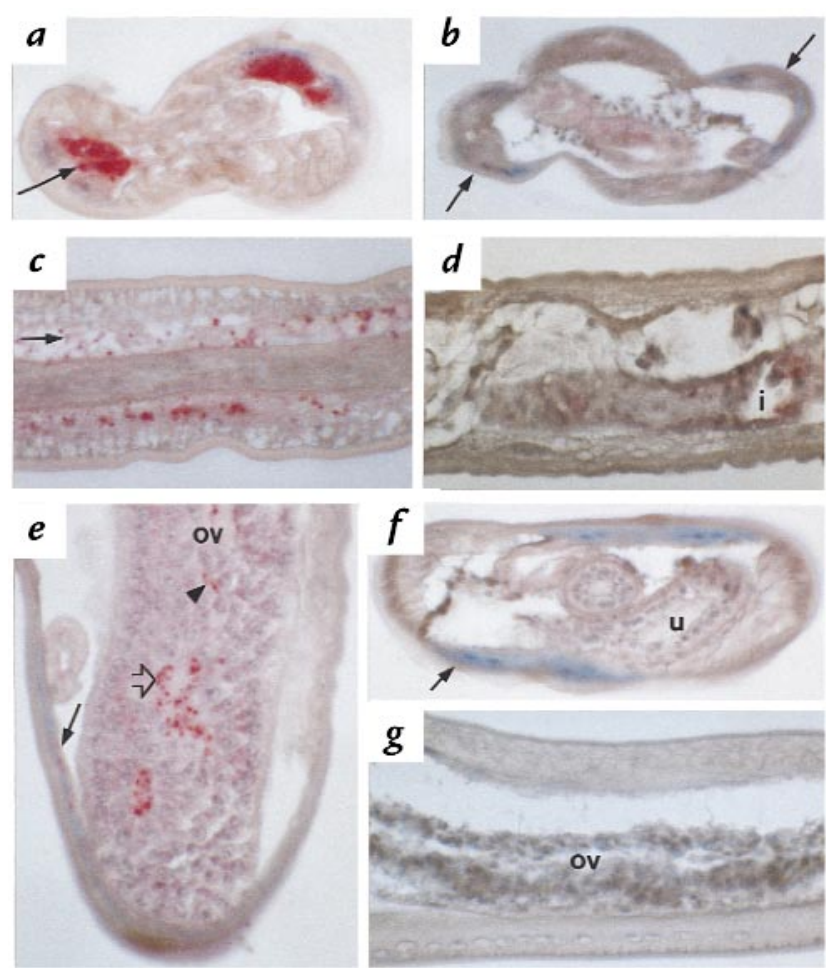

Figure 2

Immunohistology of adult L. sigmodontis from BALB/c mice with or without tetracycline therapy. Staining was performed using rabbit antiserum against Onchocerca volvulus endobacterial hsp-60, APAAP. (a) Cross-section of an untreated male worm with numerous endobacteria (red) in the lateral chord of the hypodermis (arrow); day 28, $\times 800$. (b) Cross-section of a treated female worm with no endobacteria stained in the lateral chords (arrows); day $28, \times 800$. (c) Longitudinal section of an untreated female with numerous endobacteria in the hypodermis (arrow); day 28, $\times 800$. (d) Longitudinal section of a treated female with no endobacteria stained; day $28, \times 670$. (e) Oblique section of a female with endobacteria in the hypodermis (black arrow), in the rachis (white arrow) of the ovary (ov), and in oocytes (arrowhead); day 49, $\times 800$. ( $f$ ) Cross-section of a treated female with an empty uterus $(u)$ with no endobacteria stained in the hypodermis (arrow); day $42, \times 500$. (g) Longitudinal section of a treated female showing degenerated oocytes in the ovary (ov) with no endobacteria stained in the hypodermis; day $42, \times 800$. AAPAAP, alkaline phosphatase antialkaline phosphatase.

determined, and a standard curve was generated using the known concentrations of the standards. Antibiotic concentrations in sera were then calculated with the help of this standard curve after determination of the diameter of growth inhibition.

Parasitology. Microfilaremia in mice and Mastomys was determined in EDTA-treated peripheral blood after staining with Hinkelmann's solution $(0.5 \% \mathrm{wt} / \mathrm{vol}$ eosin $\mathrm{Y}, 0.5 \% \mathrm{wt} / \mathrm{vol}$ phenol, and $0.185 \mathrm{vol} / \mathrm{vol}$ formaldehyde in distilled water). Three hundred microliters of Hinkelmann's solution was mixed with $50 \mu \mathrm{l}$ EDTA-treated peripheral blood and incubated for $2 \mathrm{~h}$ at room temperature. The suspension was then centrifuged at $2000 \mathrm{~g}$, and the pellet resuspended in $10-\mu \mathrm{l}$ residual supernatant. Microfilariae were counted in a Neubauer cytometer.(Brand GmbH, Wertheim, Germany) Microfilaremia in cotton rats was determined by mixing $10 \mu$ EDTA-treated peripheral blood with $40 \mu \mathrm{l}$ of a $3 \%$ acetic acid and $0.02 \%$ gentian violet solution, and counting in a Neubauer cytometer.

For examination of adult $L$. sigmodontis nematodes, animals were sacrificed at the ends of the respective experiments, and the pleural and peritoneal cavities were flushed with PBS-1\% FCS. Adult A. viteae were dissected from the subcutaneous and muscular tissues of multimammate rats. After length determination, adult female nematodes were placed on glass slides and examined under a dissecting microscope for the presence of undelivered motile microfilariae. The extent of embryogenesis was determined by subjecting nematodes to squeeze preparation after fixation in $80 \%$ ethanol (22). Statistical analysis of the differences in nematode burdens and nematode length was carried out using the Student's $t$ test. Animal trials were approved by the Review Board of the Bernhard Nocht Institute.

Electron microscopy. Filariae were fixed in $2 \%$ cacodylate buffered glutaraldehyde, postfixed in $1 \%$ osmium tetroxide, treated with $1 \%$ tannic acid, dehydrated in ethanol and propylene oxide, and embedded in epoxy resin. Semithin plastic sections were cut with glass knives with a Reichert-Jung Ultra Cut E (Leica, Bensheim, Germany) and stained with the combined methylene-blue azure II for light microscopy. Ultrathin sections were cut with diamond knives, stained with uranyl acetate and lead citrate, and examined with a Philips CM 10 (Philips, Eindhoven, the Netherlands).

Immunohistology. Juvenile and adult worms were fixed either in $80 \%$ ethanol or $4 \%$ cold buffered formaldehyde solution and embedded in paraffin using standard methods. For immunostaining, the alkaline phosphatase antialkaline phosphatase technique was applied according to the recommendations of the manufacturer (DAKO Corp., Hamburg, Germany). A rabbit antiserum against the recombinant heat shock protein-60 (hsp-60) of the intracellular bacteria in the human filaria $O$. volvulus (23) and the preimmune serum of the same rabbit were from the laboratory of M. Gallin and K.D. Erttmann (Bernhard Nocht Institute). A rabbit antiserum against hsp-60 of Yersinia enterocolitica (24) was a kind gift of I. Autenrieth (University of Munich, Munich, Germany). Rabbit antisera were used as primary antibodies at dilutions of 1:500 and 1:1,000. Anti-rabbit polyclonal antibody (DAKO Corp.) was used as secondary antibody. Fast red TR salt (Sigma-Aldrich Chemie GmbH, Deisenhofen, Germany) was used as the chromogen, and hematoxylin (Merck, Darmstadt, Germany) served as the counterstain.

Isolation of endobacterial $16 S$ gene from L. sigmodontis genomic $D N A$. Adult worms were homogenized in lysis buffer $(50 \mathrm{mM}$ Tris-HCl, pH 8.0; 20 mM EDTA, pH 8.0; 2\% SDS) with a glassin-glass homogenizer. Subsequently, $0.1 \mathrm{vol}$ of $10 \mathrm{mg} / \mathrm{ml}$ proteinase $\mathrm{K}$ was added to the parasite extract, which was then incubated for $30 \mathrm{~min}$ at $37^{\circ} \mathrm{C}$. The DNA was extracted twice with phenol/chloroform, once with chloroform, followed by ether extraction and ethanol precipitation.

Polymerase chain reactions with the endobacterial primers described here were performed on DNA samples from adult L. sigmodontis from mice and cotton rats. The primers used were either universal eubacterial $16 \mathrm{~S}$ primers EUBAC-1 (5'AGAGTTTGATCCTGGCTCAG- $\left.3^{\prime}\right)$ and EUBAC-2 (5'-AAGGAGGTGATCCAGCC-3') (25), or filarial endobacterial primers FIL-1 (5'-TATATAGCTTGCTATAGTGTA-3') and FIL-2 (5'TCGAACAGGCATAATTTCCA-3') (9). The expected PCR products with the EUBAC-1/EUBAC-2 primer pair and with the FIL1/FIL-2 primer pair are 1,483 and $898 \mathrm{bp}$, respectively. Negative controls without DNA were always included in parallel. The nucleotide sequences of the PCR fragments were determined by standard dideoxy-chain termination reactions on double-stranded DNA, using ${ }^{35} \mathrm{~S}$-dATP. The nucleotide sequence has been submitted to GenBank and has the accession No. AF069068.

Phylogenetic analyses. 16S sequences from 15 species of the $\alpha$ proteobacteria (see Fig. $3 b$; sequences have their GenBank accession numbers, with species binomen or the host binomen with "es" prefixed), including seven Wolbachia-like endobacteria from the wasp genus Nasonia spanning both groups of Wolbachia-like endobacteria (see Fig. 3, groups $A$ and B) $(26,27)$ were aligned and subjected to phylogenetic analy- 
sis using maximum parsimony (MP) and minimum evolution (neighbor joining $[\mathrm{NJ}]$ with logDet transformation) (28-30). These 16 taxa were chosen as being representative of $160 \alpha$ proteobacterial $16 \mathrm{~S}$ sequences in the database. Of 1,045 aligned characters, 961 are constant and 362 informative for MP. Statistical support for the phylogenies retrieved was estimated using the bootstrap resampling procedure, with 100 (MP) or 1,000 (NJ) replicates.

\section{Results}

Localization of endobacteria in L. sigmodontis. In the strain of L. sigmodontis used for this study, bacteria were readily detectable with transmission electron microscopy and found to be most abundant in the lateral chords of the hypodermis. They were also observed in the rachis of the ovary and in oocytes and embryos (Fig. 1, $a-c$ ). Using a rabbit antiserum against $O$. volvulus endobacterial hsp60 (23) for immunohistochemistry, endobacteria were seen in the hypodermis of all male and female L. sigmodontis worms collected from BALB/c mice on days 28, 49, 93, and 120 after infection, as well as in all worms from multimammate rats and cotton rats. They were usually most numerous in the lateral chords of the hypodermis (Fig. 2, a, c, and e), as well as in the rachis and the oocytes in the ovary (Fig. 2e), and in developing embryos. No positive staining was observed at these sites using preimmune serum. The same staining pattern (data not shown) was also observed with a rabbit antiserum against hsp-60 of Y. enterocolitica (24).

Relationship of L. sigmodontis intracellular bacteria to the genus Wolbachia endobacteria of arthropods. A 1,483-bp fragment of the bacterial 16S ribosomal RNA gene was amplified from genomic DNA prepared from L. sigmodontis and sequenced on both strands (GenBank accession no. AF069068). The sequence was most similar to $16 \mathrm{~S}$ genes from the Wolbachia group of intracellular bacteria, including those recently described from endobacteria of D. immitis (9) and O. volvulus (31). The filarial endobacterial 16S sequences were aligned to $16 \mathrm{~S}$ genes from Wolbachia and other members of the Rickettsiales (part of the proteobacteria $\alpha$ subdivision of Eubacteria), including Ehrlichia, Cowdria, and Rickettsia species. Analysis of this alignment with MP (Fig. 3) and minimum evolution (NJ, not shown) algorithms (30) revealed that the filarial endobacteria form a strongly supported clade $(100 \%$ bootstrap support) with the arthropod endobacteria (Wolbachia species), distinct from other Rickettsiales. In addition, the sequence divergence between the filarialderived $16 \mathrm{~S}$ sequences is much greater than that seen within either A or B group of arthropod Wolbachia. This indicates that the filarial Wolbachia species form distinct clade(s) (non-A, non-B) and argues for vertical transmission within filarial nematodes and against their recent spread from arthropod vectors. The sequences analyzed do not yield a strongly supported branching order for the filarial and arthropod clades. Neighbor joining gives high bootstrap support for a filarial endobacteria clade (71\%), but this is not evident when the sequences are analyzed using MP (maximal bootstrap support, 57\%).

Elimination of filarial endobacteria by tetracycline therapy of L. sigmodontis-infected mice. The close relationship of the endobacteria of L. sigmodontis to the genus Wolbachia prompted us to investigate the beneficial potential of tetracycline therapy. Tetracycline, injected into BALB/c mice intraperitoneally daily at a dose of $50 \mathrm{mg} / \mathrm{kg} /$ day (1 $\mathrm{mg} / \mathrm{animal} /$ day), resulted in a maximal serum concentration of this antibiotic of $8.3 \pm 0.8 \mu \mathrm{g} / \mathrm{ml}$ (Table 1 ), approximately eightfold the minimum inhibitory concentration (MIC) calculated for Rickettsiales (32). When nematodes obtained from mice treated with tetracycline from days 1 to 28 after infection were analyzed by transmission electron microscopy, intact bacteria were absent and few structures resembling remnants of bacteria were detectable (Fig. 1d). Consistent with these electron microscopic findings, no expression of the bacterial hsp-60 was observed by immunohistology in any filariae from tetracycline-treated mice collected at day 28 after infection (Fig. 2, $b$ and $d$ ). The same pattern was observed in worms from mice that had been treated from day 1 until day 42 (Fig. 2, $f$ and $g$ ) or day 63 after infection.

Infertility of adult female filariae induced by tetracycline therapy. Tetracycline therapy of L. sigmodontis-infected mice resulted in a highly significant reduction in filarial survival, size, and fertility. By 28 days after infection (i.e., after the period of development of infective larvae to young adult worms [18]), tetracycline-treated mice harbored only $60 \%$ of the number of adult worms compared with infected, untreated controls (Table 2). The remaining worms in tetracycline-treated mice displayed a highly retarded growth (Table 2 ). At day 42 after infec-

Table 2

Effect of tetracycline treatment on the development of L. sigmodontis in BALB/c mice

\begin{tabular}{|c|c|c|c|c|c|c|}
\hline \multirow[t]{2}{*}{ Group of BALB/c } & \multirow{2}{*}{$\begin{array}{l}\text { Adult worm } \\
\text { load/mouse } \\
\text { (28 days p.i.) }\end{array}$} & \multicolumn{3}{|c|}{ Female worm length $(\mathrm{mm})$} & \multirow[b]{2}{*}{$\begin{array}{l}\text { No. of mice } \\
\text { patent/total }\end{array}$} & \multirow[b]{2}{*}{$\begin{array}{l}\text { Median microfilaremia } \\
\quad(\mathrm{mf} / 50 \mu \mathrm{l} \text { blood })\end{array}$} \\
\hline & & $\begin{array}{c}\text { Juvenile } \\
\text { (28 days p.i.) }\end{array}$ & $\begin{array}{c}\text { Adult } \\
\text { (42 days p.i.) }\end{array}$ & $\begin{array}{c}\text { Adult } \\
\text { (63 days p.i.) }\end{array}$ & & \\
\hline $\begin{array}{l}\text { Infected, treated with } \\
\text { tetracycline }(50 \mathrm{mg} / \mathrm{kg} / \text { day })\end{array}$ & $\begin{array}{l}17.4 \pm 13.2 \\
(n=8 \text { mice })\end{array}$ & $\begin{array}{c}6 \pm 0.5^{\mathrm{B}} \\
(n=10 \text { worms })\end{array}$ & $\begin{array}{c}7.1 \pm 0.4 \\
(n=6 \text { worms })\end{array}$ & $\begin{array}{c}16.3 \pm 2.8 \\
(n=6 \text { worms })\end{array}$ & $0 / 7$ & 0 \\
\hline Infected, not treated & $\begin{array}{c}28.8 \pm 8.7 \\
(n=8 \text { mice }) \\
(P<0.025)\end{array}$ & $\begin{array}{c}16.5 \pm 2.5 \\
(n=10 \text { worms }) \\
(P<0.00001)\end{array}$ & $\begin{array}{c}40.1 \pm 3.4 \\
(n=6 \text { worms }) \\
(P<0.0001)\end{array}$ & $\begin{array}{c}75.8 \pm 10.2 \\
(n=7 \text { worms }) \\
(P<0.00001)\end{array}$ & $5 / 7$ & $13(1-93)$ \\
\hline
\end{tabular}

Data from one or two more consistent experiments are shown.

ADetermined 63 days after infection.

BWorm length in mice treated with $25 \mathrm{mg} / \mathrm{kg} /$ day: $4.9 \pm 0.7 \mathrm{~mm}(n=11$ worms $)$; in mice treated with $12.5 \mathrm{mg} / \mathrm{kg} / \mathrm{day}: 5.8 \pm 1.3 \mathrm{~mm}(n=16 \mathrm{worms})$. 
tion, the worms from tetracycline-treated mice showed degenerate oocytes (Fig. $2 g$ ), and no embryos were seen in the uterus by histology (Fig. 2f). Some worms were also disintegrating and attacked by host cells (data not shown). Application of lower doses of tetracycline per animal ( 0.5 and $0.25 \mathrm{mg} /$ animal/day) had consistent effects on filarial development (Table 2).

Under tetracycline therapy of BALB/c mice, adult female worms were infertile. In infected but untreated control mice, all stages of embryogenesis could be detected, from oocytes to pretzel stages and motile microfilariae. In worms from tetracycline-treated mice, pretzel stages and microfilariae were lacking, and the anterior parts of the worms' uteri usually harboring those later stages of microfilarial development were empty (Fig. 2f). In control mice, patent microfilaremia was observed from weeks 8 to 15 , as seen previously (18), but was absent in tetracycline-treated animals (Table 2).

Unaltered nematode development after treatment with antibiotics not or moderately affecting rickettsial bacteria. Antibiotics known (33) to be ineffective in eliminating Rickettsiales (penicillin G, gentamicin) or less effective than tetracycline against some species (ciprofloxacin) were used to treat L. sigmodontis-infected mice from days 1 to 63 after infection as a control for tetracycline therapy. Although peak serum concentrations of these antibiotics were clearly above the MIC (Table 1), none of these treatments eliminated bacteria from L. sigmodontis in their murine hosts (not shown). Consistent with a mutualistic interaction between endobacteria and worms, the normal development of worms in BALB/c mice treated with these antibiotics was not altered: these worms displayed normal length (gentamicin: $71.8 \pm 13.3 \mathrm{~mm}$; penicillin: $65.0 \pm 6.4 \mathrm{~mm}$; ciprofloxacin: $70.0 \pm 11.3 \mathrm{~mm}$ ) and fertility at day 63 after infection.

Unaltered development of endobacteria-devoid A. viteae after tetracycline treatment. To further elucidate the association between endobacteria and normal filarial development, tetracycline treatment was applied to experimental infection of multimammate rats with the filarial nematode A. viteae (20), a filarial species in which lack of endobacteria was reported (5) and confirmed by us using electron microscopy and immunohistology (not shown). Application of tetracycline ( $25 \mathrm{mg} / \mathrm{kg} /$ day) to A. viteae-infected multimammate rats (the easiest-tohandle permissive rodent, given that laboratory mice are nonpermissive for this parasite) from days 1 to 70 after infection did not alter the development and fertility of
A. viteae (Table 3). In contrast, tetracycline treatment of L. sigmodontis infections in multimammate rats (as a control for effects of the antibiotic on the host) was equivalent (not shown) in efficacy to that described for BALB/c mice (Table 2).

Beneficial effect of tetracycline on host microfilarial load after onset of patency. In L. sigmodontis infection of the natural host, the cotton rat, animals become microfilaremic three to four months after infection and display a high microfilaremia (>4,000 mf/ $\mu \mathrm{l}$ blood) that lasts for more than six months with only a slow decrease (unpublished observations), in contrast to BALB/c mice (only three weeks of microfilaremia). Therefore, cotton rats instead of BALB/c mice were used to assess the impact of tetracycline (added at $0.3 \%$ final concentration to the drinking water) on microfilarial load when treatment was initiated subsequent to the onset of patency, but before microfilarial load had peaked. In 150-day-infected but untreated cotton rats, a normal course of patency (microfilaremia) was observed (Fig. 4). In contrast, in 150-day-infected cotton rats treated with tetracycline, there was a significant drop in microfilaremia within a month of the initiation of therapy. After 41 days of therapy, microfilaremia in tetracycline-treated animals was only one-tenth that in infected, untreated rats (Fig. 4), and only 21 of 64 (33\%) female $L$. sigmodontis from tetracycline-treated rats harbored live, motile microfilariae, compared with 43 of 54 (80\%) worms from infected, untreated rats. These data demonstrate that tetracycline treatment of parasitized hosts is also beneficial if initiated subsequent to the onset of patency.

\section{Discussion}

Intracellular bacteria were first described in filarial nematodes more than 20 years ago (5). Although these bacteria have sometimes been termed endosymbionts based on their intimate association with their filarial hosts (9), neither the nature of this association (parasitic, neutral, or mutually beneficial) nor the effects of bacterial elimination on worm development have been elucidated. Previously, a beneficial effect of tetracycline therapy in jirds infected with Brugia pahangi was reported, but the mechanism of the effect was not analyzed with respect to endobacterial biology (34).

This study shows for the first time that the antibiotic tetracycline is able to eliminate endobacteria when administered to the mammalian host (Figs. 1 and 2), implying that the antibiotic must be able to penetrate host and worm tissues. Depletion of bacteria from L. sigmodontis by tetracycline therapy of infected rodent hosts is strictly

Table 3

Effect of tetracycline treatment on the development of Acanthocheilonema viteae in Mastomys natalensis

\begin{tabular}{|c|c|c|c|c|c|c|}
\hline \multirow[t]{2}{*}{ Treatment } & \multirow[t]{2}{*}{ No. of Mastomys patent/total } & \multirow[t]{2}{*}{ Microfilaremia $(\mathrm{mf} / \mu \mathrm{l}$ blood) } & \multicolumn{2}{|c|}{ No. of worms/Mastomys } & \multicolumn{2}{|c|}{ Length of worms ( $\mathrm{mm})$} \\
\hline & & & Female & Male & Female & Male \\
\hline $\begin{array}{l}\text { Tetracycline } \\
(25 \mathrm{mg} / \mathrm{kg} / \text { day })\end{array}$ & $3 / 3$ & $94,187,707$ & $11.0 \pm 5.7$ & $8.3 \pm 4.1$ & $\begin{array}{l}46 \pm 11 \\
(n=32)\end{array}$ & $\begin{array}{c}24 \pm 2 \\
(n=25)\end{array}$ \\
\hline None & $3 / 3$ & $42,69,894$ & $8.0 \pm 6.7$ & $7.0 \pm 4.5$ & $\begin{array}{c}48 \pm 4 \\
(n=23)\end{array}$ & $\begin{array}{c}23 \pm 3 \\
(n=19)\end{array}$ \\
\hline
\end{tabular}

All parameters were determined 70 days after infection. 


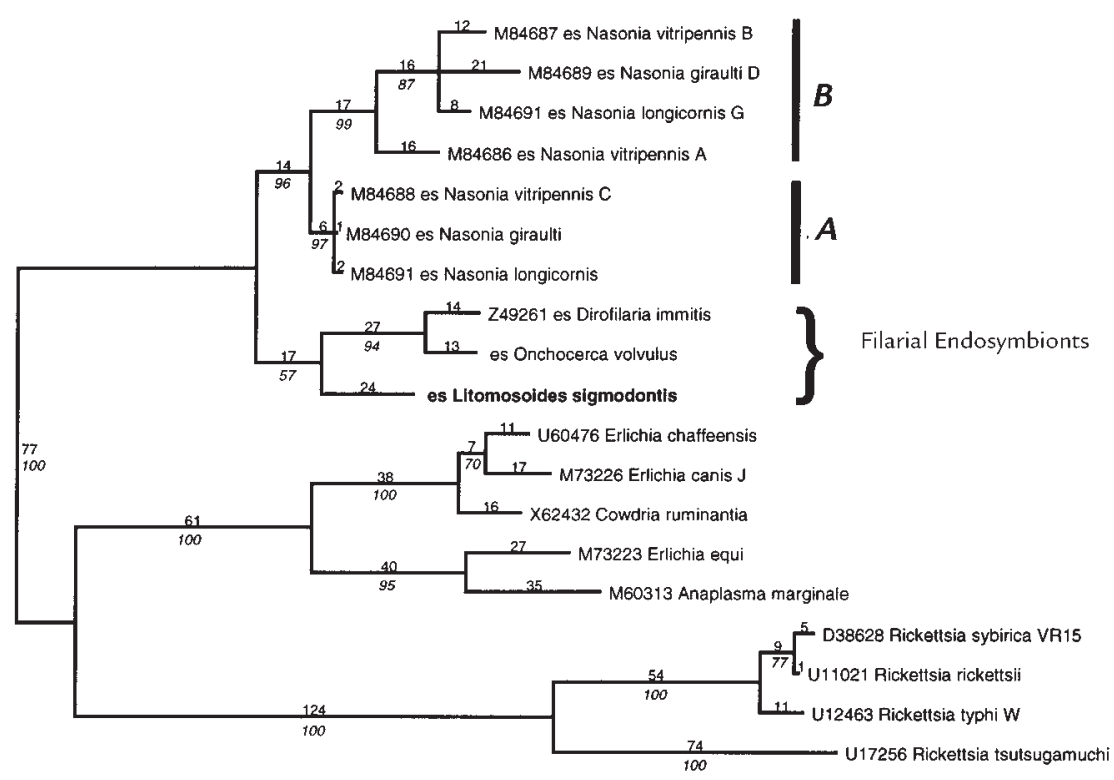

\section{Figure 3}

Phylogenetic analysis of filarial endobacterial $16 \mathrm{~S}$ rRNA gene sequences. Phylogram derived from a maximum parsimony analysis of aligned $16 \mathrm{~S}$ rRNA genes from Wolbachia species, filarial endobacteria, and representative members of the Rickettsiales. Branch lengths (inferred numbers of nucleotide changes) are given above each branch. Bootstrap support (100 replicates, heurisitic search) are given in italic below each supported branch.

associated with developmental inhibition and infertility of the nematodes (Table 2). Two effects are observed (Table 2): a block in filarial establishment and maturation (macrofilaricidal effect), and a block in embryogenesis and microfilarial production (microfilaricidal effect). Antibiotics not harmful to endobacteria, such as penicillin, gentamicin, and ciprofloxacin, do not alter the development of $L$. sigmodontis. In contrast, a strain of $A$. vitede that lacks endobacteria is not affected, despite long-lasting tetracycline therapy of the infected rodent host (Table 3).

At this time, we cannot fully exclude a pathogenic effect of killed bacteria that results in death of the treated cells and filariae. This seems unlikely, however, given that worms do not stain for bacteria after 28 days of treatment but still are alive after another 35 days of tetracycline (day 63 after infection; Table 2). Moreover, it has proved impossible, by tetracycline dose-reduction experiments, to generate worms "cured" of bacteria but still able to recover: either there was bacterial depletion and developmental arrest, or there was recovery yet in the presence of bacteria (not shown). Therefore, it is very unlikely that one could successfully perform an experiment to differentiate between damage being due to dying bacteria or due to the lack of a mutualist, i.e., transplantation of "cured" worms into a new, naive host. The aim of this type of experiment would be to establish a parasite strain devoid of bacteria; however, this would require fertility of tetracycline-treated worms, which was not seen in the absence of bacteria. In conclusion, the results on tetracycline treatment, the lack of a therapeutic "window" allowing a cure of worms from bacteria, as well as the long evolutionary conservation of these bacteria in the absence of a recent horizontal transmission, all strongly argue for a mutualistic association between endobacteria and their filarial hosts.

The filarial endobacteria are closely related to the Wolbachia endobacteria of arthropods $(9,27,35)$. The arthropod Wolbachia species can be divided into two clades, A and $\mathrm{B}$, on the basis of $16 \mathrm{~S}$ ribosomal RNA and ftsZ sequences (27). Within both of the arthropod-symbiont clades, the endobacterial species are very closely related but the phylogenetic distribution of the hosts is very wide (isopods to insects), strongly suggesting that these endobacteria have spread recently by epidemic, horizontal transfer between hosts $(26,27)$. The filarial endobacterial $16 \mathrm{~S}$ sequences are quite distinct from A and B group arthropod Wolbachia. The depth of divergence in $16 \mathrm{~S}$ sequence places the origin of the filarial endobacteria at least as ancient as the A vs. B split. A date of approximately 50 million years has been derived for the divergence of A and B group Wolbachia (35).

The sequence divergence between the filarial 16S sequences is much greater than that seen within either A or B group Wolbachia, suggesting that they have not spread recently between the nematodes by horizontal transfer. Additional $16 \mathrm{~S}$ sequence data from other filarial endobacteria (B. malayi and O. volvulus) (HenkleDührsen, K., and Blaxter, M., unpublished data) and ftsZ sequences from endobacteria of a wide range of filariae (Blaxter, M., unpublished data) confirm this analysis. The filarial endobacteria are therefore distinct Wolbachia-like lineages that are (thus far) specific to the filarial nematodes and have not been spread in recent evolutionary time from arthropod to nematode, or vice versa (Fig. 3). It remains a possibility that horizontal transfer from filariae to their arthropod vector hosts (or vice versa) has occurred at some time in the past.

To apply tetracycline as a supportive therapy to improve antifilarial treatment to humans, it will have to influence the parasite load not only when applied from the beginning of infection, before symptoms occur, but also when applied after the onset of patency, the stage at which the infection is usually diagnosed (36). In this context, it is of importance that tetracycline, when administered to animals harboring already established infections, still dramatically reduced microfilaremia (Fig. 4) and adversely affected female fertility. This could be a direct microfilaricidal effect or could result from a disruption of microfilarial production at the level of embryogenesis. Unpublished experiments from our group in which microfilariae were injected into tetracycline-treated mice do not support the first hypothesis (not shown). The gonadal 


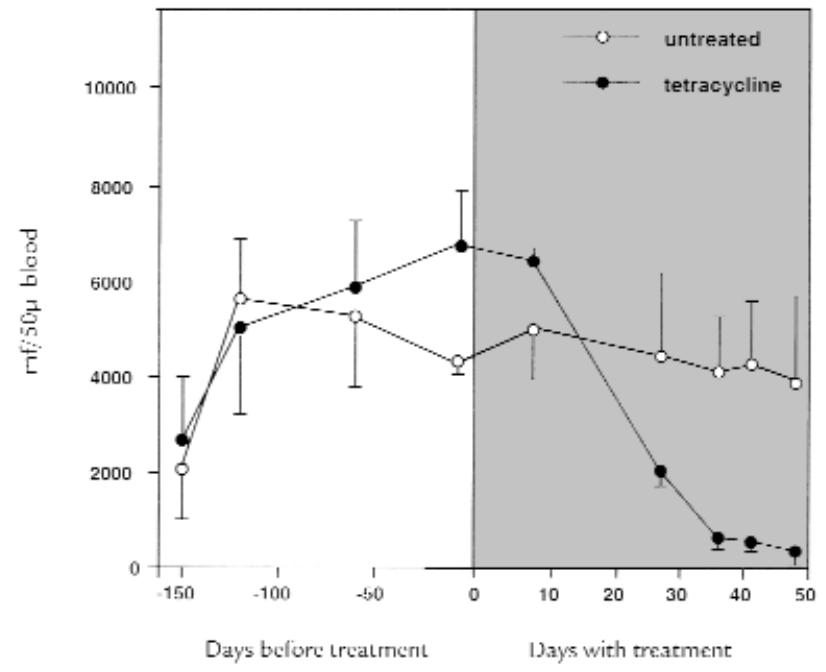

Figure 4

Effect of tetracycline treatment on the microfilaremia of $L$. sigmodontis-infected cotton rats. In contrast to infected but untreated rats, there is a quick decline in microfilaremia in rats treated with tetracycline (added at $0.3 \%$ final concentration to the drinking water).

pathology (Fig. 2g) could in turn be responsible for the developmental block. The macrofilaricidal and antimicrofilaremia effect of tetracycline during patency suggests that a similar approach might be useful for treatment of human filarial diseases. In particular, given that tetracycline affects all stages of embryonic development, it might have pharmacologic effects complementary to ivermectin, whose effects are confined to the late stage of microfilarial development, the stretched microfilariae (3), and which does not kill the endobacteria in microfilariae of O. volvulus (37). The endosymbiotic bacteria of filariae, and their potential as a target in treatment of human and animal disease, merit renewed attention.

\section{Acknowledgments}

The technical assistance of Caren Neumann and Ingeborg Albrecht is gratefully acknowledged. This study received financial support from the Edna McConnell Clark Foundation (to A. Hoerauf, K. Henkle-Dührsen, and M.L. Blaxter), the Deutsche Forschungsgemeinschaft (grant Ho 2009/1-1 to A. Hoerauf), the Darwin Trust (to M.L. Blaxter), and the German Ministry of Research and Technology (grant 01KA 9201/13 to D.W. Büttner).

1. Fiftieth World Health Assembly. 1997. Elimination of lymphatic filariasis as a public health problem. World Health Assembly. Geneva, Switzerland. 1-2.

2. World Health Organization. 1997. Chagas disease, leprosy, lymphatic filariasis, onchocerciasis: prospects for elimination. World Health Organization. Geneva, Switzerland. 11-21.

3. Albiez, E.J. et al. 1988. Histological examination of onchocercomata after therapy with ivermectin. Trop. Med. Parasitol. 39:93-99.

4. Prichard, R. 1994. Anthelmintic resistance. Vet. Parasitol. 54:259-268.

5. McLaren, D.J., Worms, M.J., Laurence, B.R., and Simpson, M.G. 1975. Micro-organisms in filarial larvae (Nematoda). Trans. R. Soc. Trop. Med. Hyg. 69:509-514.

6. Kozek, W.J., and Figueroa-M., H. 1977. Intracytoplasmic bacteria in Onchocerca volvulus. Am. J. Trop. Med. Hyg. 26:663-678.

7. Kozek, W.J. 1977. Transovarially-transmitted intracellular microorganisms in adult and larval stages of Brugia malayi.J. Parasitol. 63:992-1000.

8. Franz, M., and Büttner, D.W. 1983. The fine structure of adult Onchocerca volvulus. IV. The hypodermal chords of the female worm. Tropenmed. Parasitol. 34:122-128.
9. Sironi, M. et al. 1995. Molecular evidence for a close relative of the arthropod endosymbiont Wolbachia in a filarial worm. Mol. Biochem. Parasitol. 74:223-227.

10. Werren, J.H., Guo, L., and Windsor, D.W. 1995. Distribution of Wolbachia among neotropical arthropods. Proc. R. Soc. Lond. B Biol. Sci. 262:197-204.

11. de Bary, A. 1879. Die Erscheinung der Symbiose. Verlag von Karl J. Trübner. Leipzig-Berlin, Germany. 1-30.

12. O'Neill, S.L. 1995. Wolbachia pipientis: symbiont or parasite? Parasitol. Today. 11:168-169.

13. Min, K.-T., and Benzer, S. 1997. Wolbachia, normally a symbiont of Drosophila, can be virulent, causing degeneration and death. Proc. Natl. Acad. Sci. USA. 94:10792-10796.

14. Ewald, P. 1995. Evolution of infectious diseases. Oxford University Press. Oxford, United Kingdom. 352-368

15. Werren, J.H. 1997. Wolbachia run amok. Proc. Natl. Acad. Sci. USA. 94:11154-11155.

16. Kruppa, T.F., Iglauer, F., Ihnen, E., Miller, K., and Kunstyr, I. 1990. Mastomys natalensis or Mastomys coucha. Correct species designation in animal experiments. Trop. Med. Parasitol. 41:219-220.

17. Pichocki, R. 1967. Myopmorpha. In Grzimeks Tierleben. Vol. 11. B. Grzimek, editor. Kindler. Zurich, Switzerland. 301-397.

18. Al-Qaoud, K.M., Taubert, A., Zahner, H., Fleischer, B., and Hoerauf, A. 1997. Infection of BALB/c mice with the filarial nematode Litomosoides sigmodontis: role of $\mathrm{CD}^{+} \mathrm{T}$ cells in controlling larval development. Infect. Immun. 65:2457-2461.

19. Al-Qaoud, K.M., Fleischer, B., and Hoerauf, A. 1998. The Xid defect imparts susceptibility to experimental murine filariosis - association with a lack of antibody and IL-10 production by B cells in response to phosphorylcholine. Int. Immunol. 10:17-25.

20. Lucius, R., and Textor, G. 1995. Acanthocheilonema viteae: rational design of the life cycle to increase production of parasite material with less experimental animals. Appl. Parasitol. 36:22-33.

21. Koneman, E.W., Allen, S.D., Janda, W.M., Schreckenberger, P.C., and Winn, W.C. 1997. Color atlas and textbook of diagnostic microbiology. Lippincott-Raven Publishers. Philadelphia, PA/New York, NY. 846 pp.

22. Schulz-Key, H. 1988. The collagenase technique: how to isolate and examine adult Onchocerca volvulus for the evaluation of drug effects. Trop. Med. Parasitol. 39:423-440.

23. Gallin, M., Koszarski, A., Büttner, D., and Erttmann, K. 1998. Cloning and characterization of a novel heat-shock protein 60 (hsp 60) associated with intracellular bacteria of the human filarial nematode Onchocerca volvulus. Nucl. Acid Res. In press.

24. Noll, A., Roggenkamp, A., Heesemann, J., and Autenrieth, I.B. 1994. Protective role for heat shock protein-reactive alpha beta $T$ cells in murine yersiniosis. Infect. Immun. 62:2784-2791.

25. Weisburg, W.G., Barns, S.M., Pelletier, D.A., and Lane, D.J. 1991. 16S ribosomal DNA amplification for a phylogenetic study. J. Bacteriol. 173:697-703.

26. Breeuwer, J.A. et al. 1992. Phylogeny of cytoplasmic incompatibility micro-organisms in the parasitoid wasp genus Nasonia (Hymenoptera: Pteromalidae) based on $16 \mathrm{~S}$ ribosomal DNA sequences. Insect Mol. Biol. 1:25-36.

27. Werren, J.H., Zhang, W., and Guo, L.R. 1995. Evolution and phylogeny of Wolbachia: reproductive parasites of arthropods. Proc. R. Soc. Lond. B Biol. Sci. 261:55-71.

28. Swofford, D.L. 1993. PAUP: phylogenetic analysis using parsimony, Version 3.1. Illinois Natural History Society. Champaign, IL.

29. Kumar, S., Tamura, K., and Nei, M. 1993. MEGA: molecular evolutionary genetics analysis, Version 1.0. Pennsylvania State University. Philadelphia, PA.

30. Blaxter, M.L. et al. 1998. A molecular evolutionary framework for the phylum Nematoda. Nature. 392:71-75.

31. Henkle-Dührsen, K., Eckelt, V., Wildenburg, G., Blaxter, M., and Walter, R.D. 1998. Gene structure, activity and localization of a catalase from intracellular bacteria in Onchocerca volvulus. Mol. Biochem. Parasitol. In press

32. Sahm, D.F., and Washington, J.A. 1991. Antibacterial susceptibility tests: dilution methods. In Manual of clinical microbiology. A. Balows, editor. American Society for Microbiology. Washington, DC. 1105-1116.

33. Raoult, D., and Drancourt, M. 1991. Antimicrobial therapy of rickettsial diseases. Antimicrob. Agents Chemother. 35:2457-2462.

34. Bosshardt, S.C. et al. 1993. Prophylactic activity of tetracycline against Brugia pahangi infection in jirds (Meriones unguiculatus). J. Parasitol. 79:775-777.

35. Werren, J.H. 1997. Biology of Wolbachia. Ann. Rev. Entomol. 42:587-609. 36. McMahon, J.E., and Simonsen, P.E. 1996. Filariases. In Manson `stropical diseases. G.C. Cook, editor. W.B. Saunders. London, United Kingdom. $1321-1368$.

37. Darge, K., Lucius, R., Monson, M.H., Behrendsen, J., and Büttner, D.W. 1991. Immunohistological and electron microscopic studies of microfilariae in skin and lymph nodes from onchocerciasis patients after ivermectin treatment. Trop. Med. Parasitol. 42:361-367. 